\title{
Effect of Oxygen on the Electronic Band Structure in $\mathrm{ZnO}_{\mathrm{x}} \mathrm{Se}_{1-\mathrm{x}}$ Alloys
}

\author{
W. Shan, ${ }^{a}$ W. Walukiewicz, J.W. Ager III, K.M. Yu, and J. Wu \\ Materials Sciences Division, Lawrence Berkeley National Laboratory, Berkeley, CA 94720 \\ E. E. Haller \\ Materials Sciences Division, Lawrence Berkeley National Laboratory, and Department of \\ Materials Science and Engineering, University of California, Berkeley, CA 94720 \\ Y. Nabetani, T. Mukawa, Y. Ito, and T. Matsumoto \\ Department of Electrical Engineering, University of Yamanashi, Kofu 400-8511, Japan
}

The effect of alloying small amounts of $\mathrm{ZnO}$ with $\mathrm{ZnSe}$ on the electronic band structure has been studied. Optical transitions in MBE-grown $\mathrm{ZnO}_{x} \mathrm{Se}_{1-\mathrm{x}}$ epitaxial films $(0 \leq x \leq 1.35 \%)$ were investigated using photoreflectance and photoluminescence spectroscopies. The fundamental band-gap energy of the alloys was found to decrease at a rate of about $0.1 \mathrm{eV}$ per atomic percent of oxygen. The pressure dependence of the band gap was also found to be strongly affected by the $\mathrm{O}$ incorporation. Both the effects can be quantitatively explained by an anticrossing interaction between the extended states of the conduction band of $\mathrm{ZnSe}$ and the highly localized oxygen states located at approximately $0.22 \mathrm{eV}$ above the conduction band edge.

PACS numbers: 71.20.-b, 71.20.Nr, 72.80.Ey, 78.20.-e

a) Electronic mail address: WShan@lbl.gov 
Highly mismatched semiconductor alloys (HMAs) in which the constituent anion elements is replaced by an element with highly dissimilar properties are currently attracting considerable attention. The electronic properties of HMAs deviate drastically from the predictions of the virtual crystal approximation (VCA). ${ }^{1,2}$ The most extensively studied HMAs to date are III-N-V alloy systems. It has been found that the substitution of the group V element in group III-V compounds with small amounts of nitrogen leads to dramatic changes of the electronic properties, resulting in a reduction of the fundamental band-gap energy as opposed to the increase predicted by the $\mathrm{VCA},{ }^{3,4}$ significant increase in electron effective mass and decrease in electron mobility, ${ }^{5-7}$ and the appearance of a new optical transition $\left(E_{+}\right)$from the valence band to the conduction band at the $\Gamma$ point. ${ }^{8,9}$ As one quantitative example, the incorporation only one percent of nitrogen into GaAs can induce a strikingly large reduction of $0.18 \mathrm{eV}$ in the fundamental band-gap energy. ${ }^{10}$

Similar effects have also been observed recently in group II-telluride compounds alloyed with Se, S, or O atoms. ${ }^{11,12}$ So far, due to the limited solubility of oxygen in II-VI compounds, the group II-O-VI alloys were synthesized by ion implantation only. A disadvantage associated with this synthesis method is that it is difficult to determine the concentration of the substitutional oxygen atoms. In this letter, we show that the recent success in growth of high quality $\mathrm{ZnO}_{\mathrm{x}} \mathrm{Se}_{1-\mathrm{x}}$ alloys using molecular beam epitaxy (MBE) makes it possible for us for the first time to carry out a systematic study of the effect of oxygen incorporation on the electronic band structure of this representative material in the group II-O-VI HMAs, as well as to derive energy position of the localized $\mathrm{O}$ state in $\mathrm{ZnSe}$ and its pressure dependence from the experimental results based a band anticrossing model. 
The $\mathrm{ZnO}_{\mathrm{x}} \mathrm{Se}_{1-\mathrm{x}}$ samples used in this study were grown on GaAs (100) substrates by molecular beam epitaxy with the growth temperature of $350^{\circ} \mathrm{C}$. Before the growth of $\mathrm{ZnOSe}$ epitaxial films, a 100-nm thick ZnSe buffer layer was deposited on the substrates. The O content in the alloy layers was controlled by varying the $\mathrm{O}_{2}$ flow rate to a $\mathrm{RF}$ oxygen plasma source. The ZnOSe layer thickness is around $600 \mathrm{~nm}$ for all the samples. The oxygen concentration in the films was determined assuming Vegard's law using lattice constants determined from the double-crystal X-ray diffraction rocking curves measured in the (400) and (511) planes. ${ }^{13}$ The uncertainty of $\mathrm{O}$ composition is about $20 \%$.

Photoreflectance (PR) and photoluminescence (PL) measurements were carried out to measure the band gap energy of the $\mathrm{ZnO}_{\mathrm{x}} \mathrm{Se}_{1-\mathrm{x}}$ samples at room temperature (295 K). For PR measurement, quasimonochromatic light from a Xenon arc lamp dispersed by a $0.5-\mathrm{M}$ monochromator was focused on the samples as a probe beam. A chopped $\mathrm{HeCd}$ laser beam (3250 ̊) provided the photomodulation. The PR signals were detected by Si photodiode using a phase-sensitive lock-in amplification system. For PL measurements, the same HeCd laser line was used for excitation and luminescence signals from the samples were dispersed by a 1-M double grating monochromator and detected by a photomultiplier tube. Application of hydrostatic pressure was accomplished by mounting small sample chips with sizes of $\sim 200 \times 200$ $\mu \mathrm{m}^{2}$ into gasketed diamond anvil cells.

Figure 1 shows PR spectra from the $\mathrm{ZnO}_{\mathrm{x}} \mathrm{Se}_{1-\mathrm{x}}$ samples used in this work. In each case, the derivative-like spectral features corresponds to the optical transitions from the valence-band edge to the conduction-band edge in the samples, and provides a direct measure of the band-gap energy. Thus, it is clear from Fig.1, the band gap of the samples shifts toward lower energy with increasing $\mathrm{O}$ concentration. The increasingly broadened PR spectral features are commonly 
observed in semiconductor alloys and can be partially attributed to the spatial fluctuation of the O composition in the sample.

Plotted in Fig. 2 are the band-gap energies of the $\mathrm{ZnO}_{\mathrm{x}} \mathrm{Se}_{1-\mathrm{x}}$ samples as a function of $\mathrm{O}$ concentration. The energy gap for each sample was determined by fitting the corresponding PR spectral curve related to the transition from the top of valence band to the bottom of the conduction band to the lineshape functional form of three-dimension interband transitions. ${ }^{14,15}$ It decreases at a rate of about $0.1 \mathrm{eV}$ per atomic percent of oxygen. This large O-induced band-gap reduction indicates a large band-gap bowing that bears a close resemblance to the analogous effects that have been extensively studied in $\mathrm{GaN}_{\mathrm{x}} \mathrm{As}_{1-\mathrm{x}}$ and $\mathrm{Ga}_{1-\mathrm{y}} \mathrm{In}_{\mathrm{y}} \mathrm{N}_{\mathrm{x}} \mathrm{As}_{1-\mathrm{x}}$ HMAs $(x<0.05)$, where the band-gap reduction caused by $\mathrm{N}$ has been successfully explained by a band anticrossing (BAC) model. ${ }^{8,16}$ Thus, we use that model here to discuss present results. The BAC model considers an anticrossing interaction of highly localized states introduced by more electronegative, isoelectronic atoms with the extended conduction-band states of a semiconductor matrix that leads to a characteristic splitting of the conduction band into two nonparabolic subbands with the energy edges given by the expression:

$$
E_{ \pm}(k)=\left(E_{O}+E_{M}(k) \pm\left[\left(E_{O}-E_{M}(k)\right)^{2}+4 C_{O M}{ }^{2} x\right]^{1 / 2}\right) / 2,
$$

where $E_{M}(k)$ and $E_{O}$ are the energies of the unperturbed conduction band edge and of the localized state relative to the top of the valence band, respectively, and $C_{O M}$ is a parameter describing the hybridization between the localized states and the extended states. The energy positions of the subband edges $E_{-}$and $E_{+}$depend on the interaction parameter $C_{O M}$ and the energetic location of $E_{O}$ with respect to the conduction band edge $E_{M}$. The solid line in the figure is the best fit using Eq.(1) to the experimental data using $C_{O M}$ and $E_{O}$ as adjustable parameters: the fit to the data is excellent. It is clear that the large reduction of the fundamental band gap in 
$\mathrm{ZnO}_{\mathrm{x}} \mathrm{Se}_{1-\mathrm{x}}$ with the increasing $\mathrm{O}$ concentration can be attributed to the downward shift of $E_{-}$ relative to the valence band as a result of band anticrossing. The theoretical fitting results yield the energy position of $\mathrm{O}$ level $E_{O}=2.90 \pm 0.02 \mathrm{eV}$ and the interaction parameter $C_{O M}=1.8 \pm 0.3 \mathrm{eV}$.

To further demonstrate that the band-gap reduction observed in the $\mathrm{ZnO}_{\mathrm{x}} \mathrm{Se}_{1-\mathrm{x}}$ samples results from the band anticrossing effect, we have also used PL to measure the energy positions of the optical transition associated with the fundamental band gap in a $\mathrm{ZnSe}_{0.9915} \mathrm{O}_{0.0085}$ sample as a function of applied hydrostatic pressure. The results are shown in Fig. 3, along with the known pressure dependence of PL features from thick ZnSe epilayers grown on GaAs substrates. ${ }^{17}$ The pressure-induced energy shifts of the optical transition related to the direct band gap of the sample is weaker and more strongly than in ZnSe. This indicates that the application of high pressure gradually changes the character of the conduction-band edge $\left(E_{-}\right)$from extended-like to localized-like. The solid line in the figure is the pressure dependence of the PL transition that corresponds to the change of the band gap of the sample calculated using Eq.(1). The best agreement with the experimental results was achieved for the energy level $E_{O}$ with a pressure dependence of $20 \mathrm{meV} / \mathrm{PGa}$. This derived pressure coefficient for the $\mathrm{O}$ level is consistent with the results of pressure dependent absorption measurements in $\mathrm{ZnTe}: \mathrm{O} .^{18}$

It is very interesting to note that the determined energy location of the $\mathrm{O}$ level in $\mathrm{ZnSe}$ can be used to evaluate band-edge offsets between $\mathrm{ZnTe}$ and $\mathrm{ZnSe}$. It has been well established that the energy levels of highly localized states are independent of the host material. For example, the locations of the d-states of transition metals, have been used to determine the band edge offsets ${ }^{19}$ and the band edge deformation potentials in compound semiconductors. ${ }^{20}$ The $\mathrm{O}$ level was previously found to locate at about $0.24 \mathrm{eV}$ below the conduction band edge in ZnTe. ${ }^{11}$ Combined this with our current result of $0.22 \mathrm{eV}$ above the conduction band edge of $\mathrm{ZnSe}$ for the 
O level, it yields a value of $\Delta E_{\mathrm{c}} \approx 0.46 \mathrm{eV}$ for the conduction-band offset and $\Delta E_{\mathrm{v}} \approx 0.9 \mathrm{eV}$ for the valence band offset for $\mathrm{ZnTe} / \mathrm{ZnSe}$ system.

Finally, it has to be pointed out that the absence of spectral features related to the optical transition associated with the conduction-band edge $E_{+}$is not inconsistent with the BAC model. Note that the $E_{+}$band edge is of mostly localized-like character, and since the dipole interaction for optical transitions couples much more strongly to extended states than localized states, the transition related to $E_{+}$is inherently weak. In addition, the energy separation between $E_{+}$and $E_{-}$ and the oscillator strength of $E_{+}$transition depend on the $\mathrm{O}$ content and the coupling parameter. In the case of $\mathrm{GaN}_{\mathrm{x}} \mathrm{As}_{1-\mathrm{x}}$, with $C_{N M}=2.7 \mathrm{eV}$, the $E_{+}$transition can be spectrally observed only at the range of $x>0.005 \sim 0.008 .{ }^{9,21,22}$ This indicates that, with an almost two times smaller coupling parameter of $C_{O M} \approx 1.8 \mathrm{eV}$ in $\mathrm{ZnO}_{\mathrm{x}} \mathrm{Se}_{1-\mathrm{x}}$, it will certainly require an alloy composition of $x>0.2$ to resolve $E_{+}$-transition related spectral feature.

In conclusion, we have studied the effect of oxygen on the electronic band structure of $\mathrm{ZnO}_{\mathrm{x}} \mathrm{Se}_{1-\mathrm{x}}$ alloys by investigating the optical transitions associated with the $\Gamma$ point at the conduction-band the valence-band edges, as well as the dependence of the transitions on applied pressure. The $\mathrm{ZnO}_{\mathrm{x}} \mathrm{Se}_{1-\mathrm{x}}$ alloy samples were found to have a significant band-gap reduction and to exhibit a classical band-anticrossing behavior in the composition and pressure dependence of the fundamental band gap. These experimental results confirmed that replacing Se anions in $\mathrm{ZnSe}$ with isoelectronic but more electronegative $\mathrm{O}$ atoms does form a highly mismatched alloy system with the properties analogous to the extensively studied III-N-V materials. The Oinduced modification of the conduction band structure can be well described using the band anticrossing model that has been successfully applied to the III-N-V alloys. 
This work is supported by the Director, Office of Science, Office of Basic Energy Sciences, Division of Materials Sciences and Engineering, of the U.S. Department of Energy under Contract No. DE-AC03-76SF00098. 


\section{References}

1. J.A. Van Vechten and T.K. Bergstresser, Phys. Rev. B1, 3351(1970).

2. R. Hill and D. Richardson, J. Phys. C4, L289(1971).

3. M. Weyers, M. Sato, H. Ando, Jpn. J. Appl. Phys. 31, L853 (1992).

4. M. Kondow, K. Uomi, K. Hosomi and T. Mozume, Jpn. J. Appl. Phys. 33, L1056 (1994).

5. C. Skierbiszewski, P. Perlin, P. Wisniewski, W. Knap, T. Suski, W. Walukiewicz, W. Shan, K.M. Yu, J.W. Ager, E.E. Haller, J.F. Geisz, and J.M. Olson, Appl. Phys. Lett. 76, 2409(2000).

6. J.F. Geisz, D.J. Friedman, J.M. Olson, S.R. Kurtz, and M.B. Keyes, J. Cryst. Growth, 195, 401(1998).

7. S.R. Kurtz, Allerman, C.H. Seager, R.M. Sieg, and E.D. Jones, Appl. Phys. Lett. 77, $400(2000)$.

8. W. Shan, W. Walukiewicz, J. W. Ager III, E. E. Haller, J. F. Geisz, D. J. Friedman, J. M. Olson, and S. R. Kurtz, Phys. Rev. Lett. 82, 1221 (1999).

9. J. D. Perkins, A. Masceranhas, Y. Zhang, J. F. Geisz, D. J. Friedman, J. M. Olson, and S. R. Kurtz, Phys. Rev. Lett. 82, 3312 (1999).

10. K. Uesugi, N. Marooka and I. Suemune, Appl. Phys. Lett. 74, 1254 (1999).

11. W. Walukiewicz, W. Shan, K.M. Yu, J.W. Ager III, E.E. Haller, I. Miotkowski, M.J. Seong, H. Alawadhi, and A.K. Ramdas, Phys. Rev. Lett. 85, 1552 (2000).

12. K. M. Yu, W. Walukiewicz, J. Wu, J. W. Beeman, J. W. Ager III, E. E. Haller, I. Miotkowski, A. K. Ramdas, and P. Becla, Appl. Phys. Lett. 80, 1571(2002).

13. Y. Nabetani, MRS Proc. Vol.744, M3.4 (2003).

14. D. E. Aspnes, in Optical Properties of Solid, edited by M. Balkanski (North-Holland, 
Amsterdam, 1980), Chap. 4A.

15. O. J. Glembocki, SPIE Proc. Vol.1286, 2(1990).

16. W. Walukiewicz, W. Shan, J. W. Ager III, D. R. Chamberlin, E. E. Haller, J. F. Geisz, D. J. Friedman, J. M. Olson, and S. R. Kurtz, in Photovoltaics for the $21^{\text {st }}$ Century, ed. V.K. Kapur, R.D. McDonnell, D. Carlson, G.P. Ceasar, and A. Rohatgi, (The Electrochemical Society, Pennington, NJ, 1999), p.199.

17. J.A. Tuchman, S. Kim, Z.F. Sui, and I.P. Herman, Phys. Rev. B46, 13371(1992).

18. W. Shan, W. Walukiewicz, J. W. Ager, and E. E. Haller, unpublished.

19. J. M. Langer and H. Heinrich, Phys. Rev. Lett, 55, 1414(1985).

20. D. D. Nolte, W. Walukiewicz, and E. E. Haller, Phys. Rev. Lett, 59, 501 (1987).

21. W. Shan, W. Walukiewicz, J. W. Ager, E. E. Haller, J. F. Geisz, D. J. Friedman, J. M. Olson, and S. R. Kurtz, J. Appl. Phys. 86, 2349(1999).

22. P.J. Klar, H. Grüning, W. Heimbrodt, J. Koch, F. Höhnsdorf, W. Stolz, P.M.A. Vicente, and J. Camassel, Appl. Phys. Lett. 76, 3439(2000). 


\section{Figure Captions:}

Figure 1. PR spectra taken from $\mathrm{ZnSe}_{1-\mathrm{x}} \mathrm{O}_{\mathrm{x}}$ samples at $295 \mathrm{~K}$. The curves are vertically displaced for clarity. The weak features near $2.7 \mathrm{eV}$ are PR signals from $\mathrm{ZnSe}$ buffer layers.

Figure 2. Band-gap energies of $\mathrm{ZnSe}_{1-\mathrm{x}} \mathrm{O}_{\mathrm{x}}$ samples as a function of oxygen concentration. The solid line is the best fit using the BAC model.

Figure 3. The effect of applied pressure on the energy of optical transitions associated with the

direct band gap of a $\mathrm{ZnSe}_{0.9915} \mathrm{O}_{0.0085}$ sample. The solid line is the calculated result using the BAC model. 


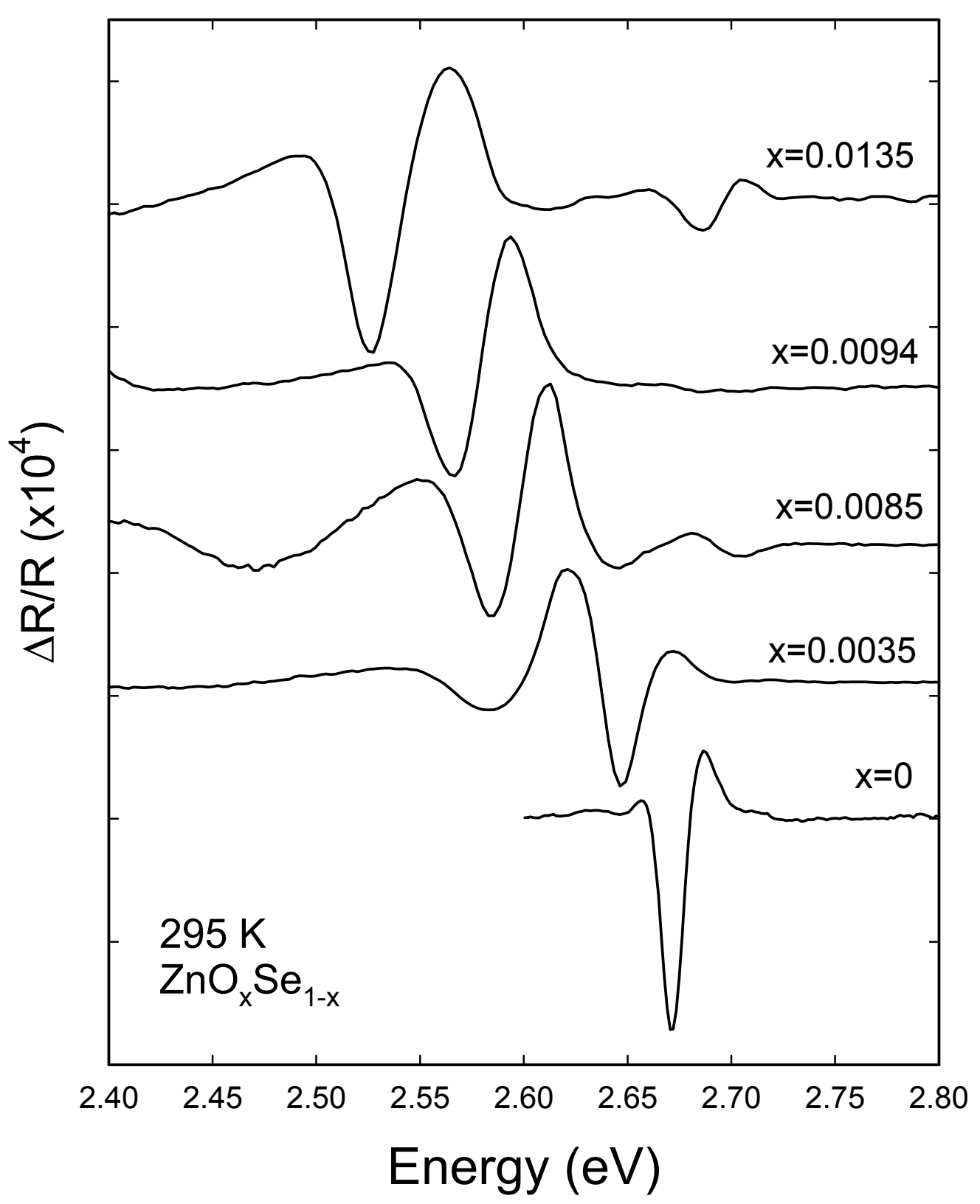

Figure 1. 


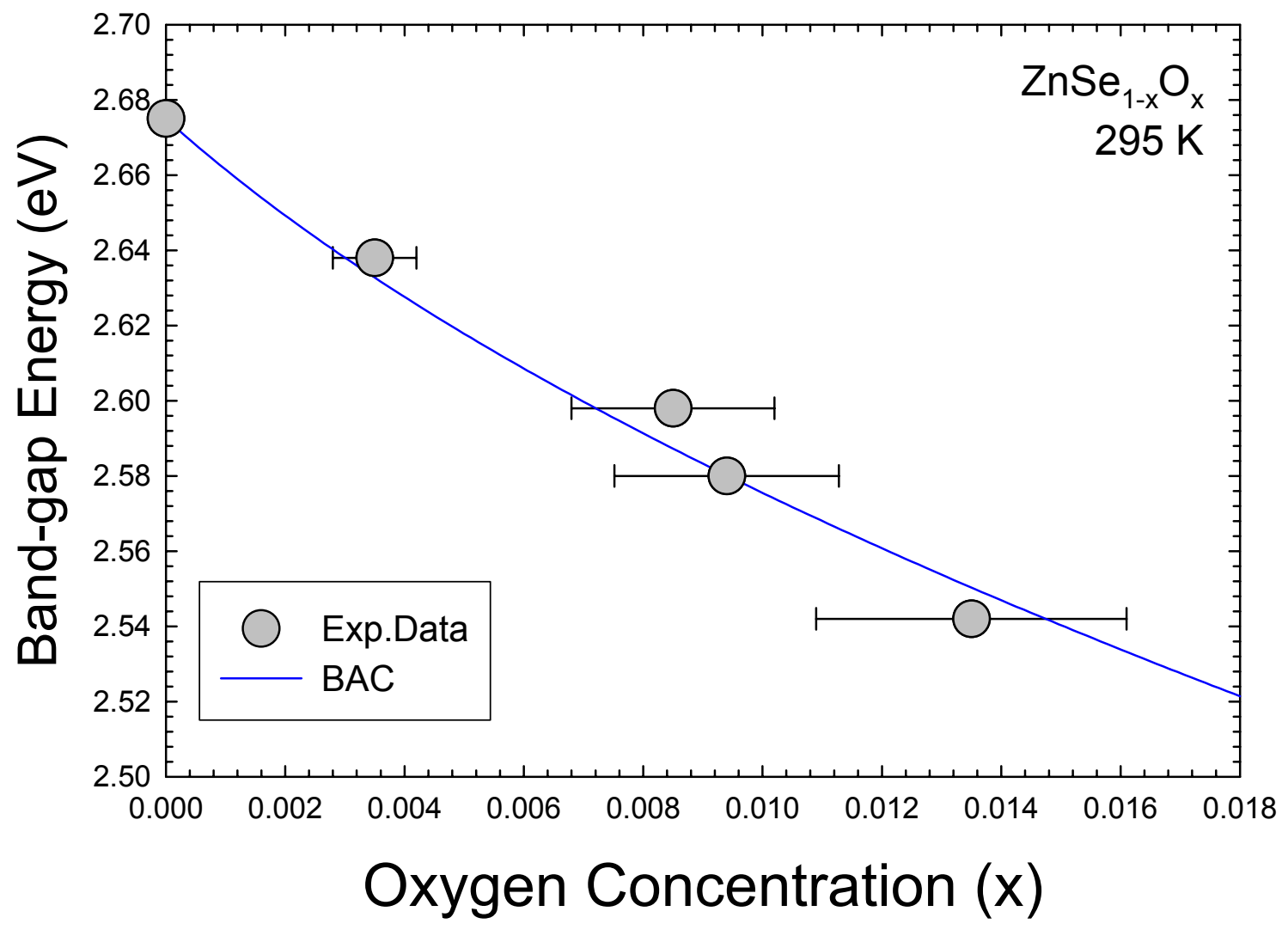

Figure 2 


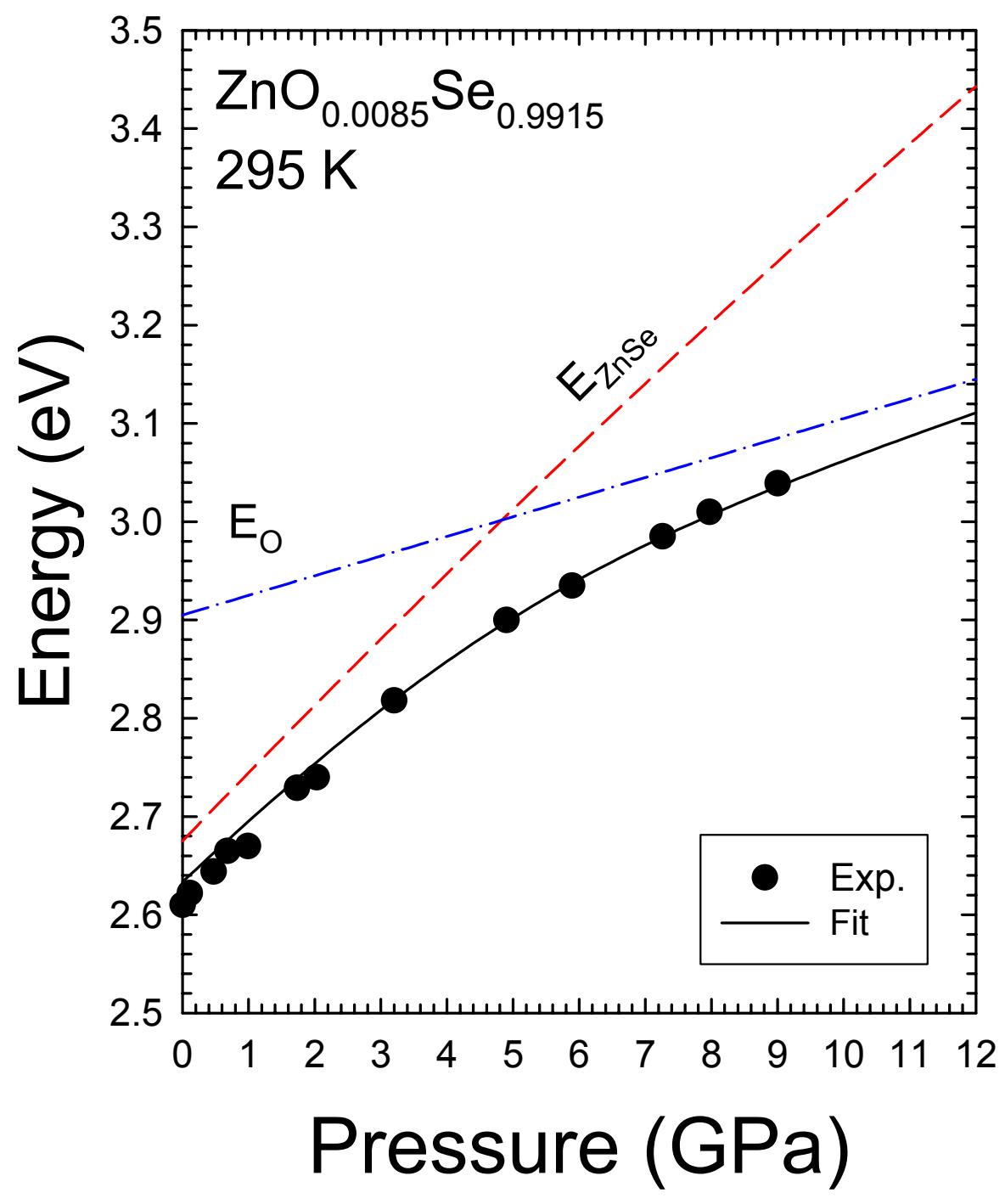

Figure 3 